\title{
The velocity field of UGC 6697 revisited $^{\star}$
}

\author{
G. Gavazzi ${ }^{1}$, M. Marcelin ${ }^{2}$, A. Boselli², P. Amram², J. M. Vílchez ${ }^{3}$, J. Iglesias-Paramo $^{2}$, and M. Tarenghi ${ }^{4}$ \\ 1 Università degli Studi di Milano - Bicocca, P.zza delle scienze 3, 20126 Milano, Italy \\ 2 Laboratoire d'Astrophysique de Marseille, 2 place Le Verrier, 13248 Marseille Cedex 4, France \\ 3 Instituto de Astrofísica de Andalucía, CSIC, Apdo. 3004, 18080, Granada, Spain \\ 4 European Southern Observatory, K. Schwarzschild Str. 2, 857448, Garching bei Muenchen, Germany
}

Received 21 December 2000 / Accepted 5 July 2001

\begin{abstract}
The edge-on galaxy UGC 6697 has a peculiar morphological appearance: a high surface brightness SE component and what appears to be a tidal tail with blue color and low surface brightness extending toward the NW, as dramatically evidenced in subarcsecond broad band images taken with the VLT and in a deep $\mathrm{H}_{\alpha}$ frame. Long-slit spectroscopy shows a $500 \mathrm{~km} \mathrm{~s}^{-1}$ rigid-body rotation curve with a sudden central velocity jump, of more than $200 \mathrm{~km} \mathrm{~s}^{-1}$ amplitude. Fabry-Perot observations of the $\mathrm{H}_{\alpha}$ emission line enable us to trace a detailed $2 \mathrm{D}$ velocity field, confirming the complex kinematical behaviour, particularly in the circumnuclear region where multiple velocity components are clearly superposed on the same line of sight. This region hosts a double nucleus and shows sudden color and metallicity gradients, which can be reconciled with the complex velocity field assuming the presence of a second galaxy hidden behind the main body of UGC 6697 .
\end{abstract}

Key words. galaxies: individual: UGC 6697 - galaxies: interactions - kinematics and dynamics

\section{Introduction}

UGC 6697 (CGCG 97-087) is a bright Irregular edgeon galaxy near the center of the cluster A1367. The attention on this object was first focused because of the bright (Gavazzi 1978) and asymmetrically extended radio source associated with it (Gavazzi \& Jaffe 1985). More recent radio continuum observations (Gavazzi et al. 1984, herafter G84; Gavazzi \& Jaffe 1987), confirmed the "head-tail" appearence of the radio source. Meanwhile $21 \mathrm{~cm}$ line measurements (Gavazzi 1989; Dickey \& Gavazzi 1991) revealed that the galaxy has a nearly normal HI content, distributed however asymmetrically with respect to the galaxy center, as opposed to its $\mathrm{H}_{2}$ content which appears normal in all respects (Boselli et al. 1994). Detailed surface photometry of the source at optical and NIR wavelengths was gathered by Gavazzi et al. (1995; hereafter G95). An early dynamical study of this object was carried out with long-slit intermediate dispersion spectroscopy at the ESO/3.6 m telescope (G84).

Send offprint requests to: G. Gavazzi, e-mail: Giuseppe.Gavazzi@mib.infn.it

* Based on observations taken at the ESO/VLT, INT, OHP, CFHT and Loiano telescopes. The INT is operated by the I.N.G. at the Spanish Observatorio del Roque de Los Muchachos of the I.A.C. The OHP is operated by the French CNRS. The Loiano telescope belongs to the University of Bologna (Italy).
The spectrum showed a rigid-body rotation curve, expected for such highly inclined system, with a maximum rotational velocity $\Delta V_{\max } \sim 500 \mathrm{~km} \mathrm{~s}^{-1}$. Superposed to this main rotational component, however, G84 detected a remarkable high-velocity feature at $\sim 200 \mathrm{~km} \mathrm{~s}^{-1}$ near the galaxy center, that could not be univocally explained.

Altogether the plenty observational material collected so far contributed pointing out that UGC 6697 is strongly peculiar in most respects. What physical mechanisms contributed producing such disturbances remains however without a convincing interpretation.

Ram pressure stripping of the fast moving galaxy with the surrounding cluster IGM or viscous stripping (Nulsen 1982) could help explainig the "head-tail" radio appearance. Similarly the asymmetric HI distribution, combined with the marginal HI deficiency, is consistent with the ram-pressure scenario, provided however that stripping is acting since a short time (few $10^{8} \mathrm{yrs}$ ) compared with the cluster transit time, i.e. that the galaxy entered quite recently the cluster dense environment. However the rampressure mechanism does not provide a convincing explanation for the dynamical disturbances observed in the galaxy. Those call for tidal interactions, either with the cluster as a whole (Merritt 1983) or with some companion galaxy (Moore et al. 1996, 1998). There are several small diffuse objects around UGC 6697. Some of them are dwarf galaxies, such as the main companion at about 30 arcsec to the north east or the dwarf elliptical (with 


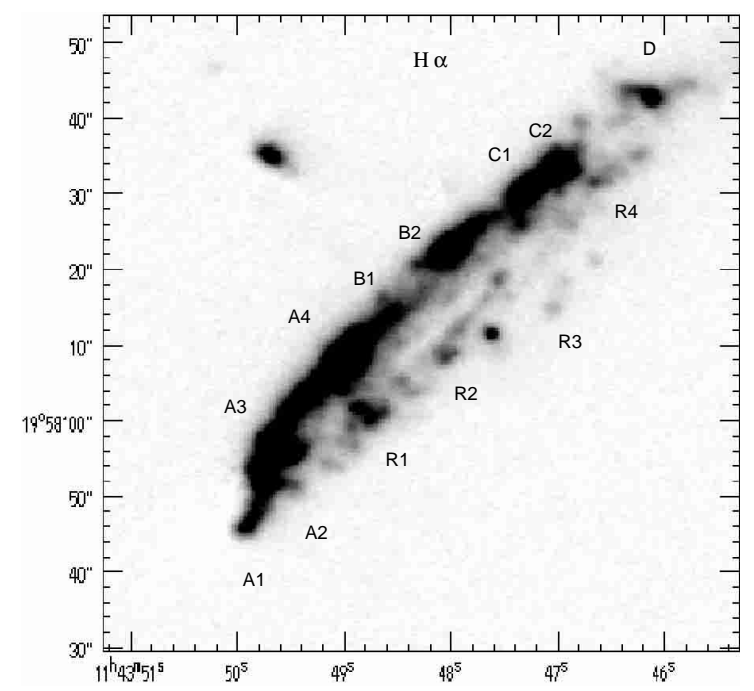

Fig. 1. The net $\mathrm{H}_{\alpha}$ INT image with enhanced contrast. The (J2000) celestial coordinates are given.

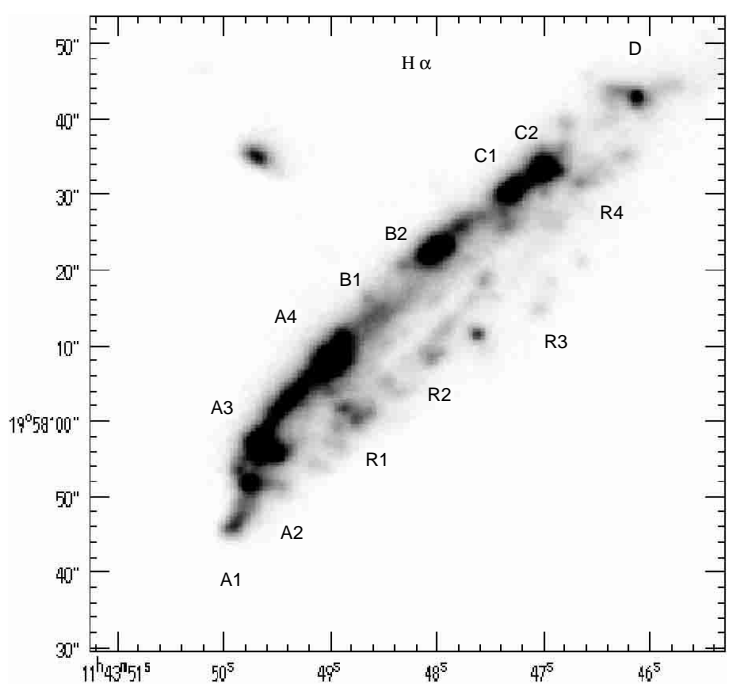

Fig. 2. The net $H_{\alpha}$ frame with shallow contrast.

no $\mathrm{H} \alpha$ emission) that can be seen close to the main body of UGC 6697 , at $\alpha=11^{\mathrm{h}} 43^{\mathrm{m}} 48.2^{\mathrm{s}} ; \delta=19^{\circ} 58^{\prime} 30^{\prime \prime}$. There are also several blobs of ionized gas with anomalous velocities (see Sects. 3.2 and 3.4) which distinguish them from the main body of UGC 6697, dramatically confirming the disturbed state of the galaxy already suggested by its morphology. The main companion is however too small and has a velocity too much different $\left(\sim 800 \mathrm{~km} \mathrm{~s}^{-1}\right)$ with respect to that of the main galaxy, and shows no signs of disturbances for being responsible for the peculiarities of UGC 6697 (see Sect. 4 for more details).

To try solving this riddle we collected more observations of UGC 6697 which we present in this paper. In particular we focused at obtaining new high resolution broadband and $\mathrm{H} \alpha$ line imaging and spectroscopy, both long-slit along several directions parallel and perpendicular to the main disk, and a 2D velocity field using Fabry-Perot interferometers.

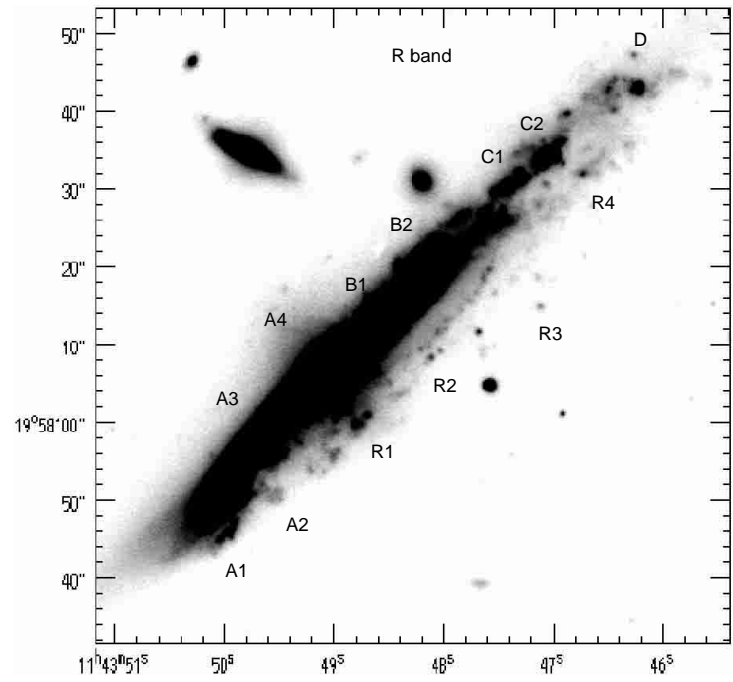

Fig. 3. The VLT $R$ frame with enhanced contrast.

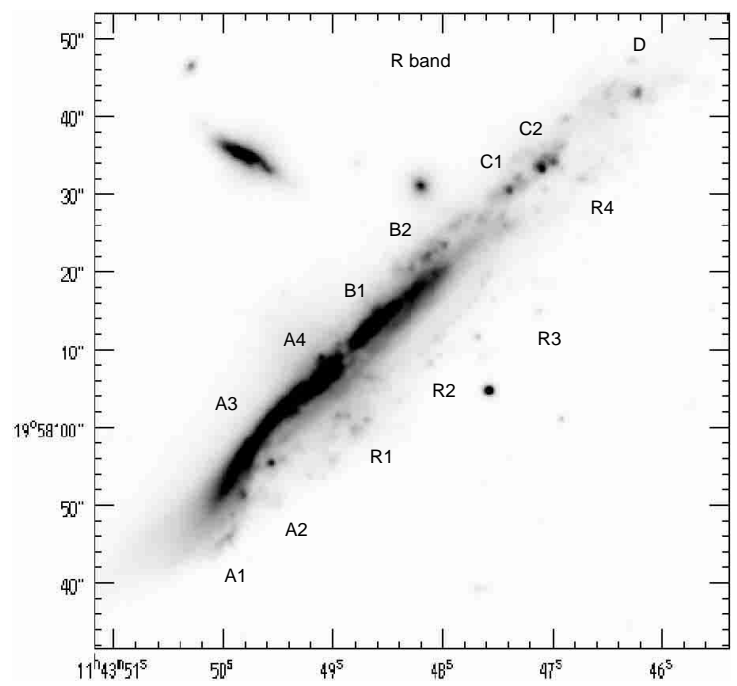

Fig. 4. The VLT $R$ frame with shallow contrast.

The presentation of the new observational material is the subject of Sect. 2, the optical morphology and the derived velocity field are given in Sect. 3 and discussed in Sect. 4 where we point out a possible explanation of UGC 6697 based on the merging with an unseen companion hidden behind the main body of UGC 6697 .

\section{Observations}

\section{1. $H_{\alpha}$ imaging}

We observed UGC 6697 through a narrow band filter centered at $6725 \AA$ ( $80 \AA$ bandpass). The image was obtained in the photometric night of April 26, 2000, in 1.5 arcsec seeing conditions, with the Wide Field Camera at the prime focus of the Isaac Newton Telescope (INT) at La Palma (see Tables 1, 3). The camera relies on 4 Chip Mosaic of thinned AR coated EEV $4 \mathrm{~K} \times 2 \mathrm{~K}$ CCDs, with pixels of $13.5 \mu \mathrm{m} \times 13.5 \mu \mathrm{m}(0.33 \operatorname{arcsec} \times 0.33 \operatorname{arcsec})$, giving a field of view of $34 \times 34$ arcmin. The ON-band 
Table 1. The imaging observations.

\begin{tabular}{lccc}
\hline Telescope Filter & $\begin{array}{c}\text { Tint } \\
\text { sec }\end{array}$ & $\begin{array}{c}\text { Seeing } \\
\text { arcsec }\end{array}$ \\
\hline VLT & $B$ & $5 \times 600$ & 1.1 \\
VLT & $V$ & $3 \times 300$ & 0.8 \\
VLT & $R$ & $3 \times 300$ & 0.7 \\
VLT & $I$ & $3 \times 300$ & 0.6 \\
INT & $\mathrm{H}_{\alpha}$ & $3 \times 1200$ & 1.5 \\
INT & $r^{\prime}$ & $3 \times 300$ & 1.5 \\
\hline
\end{tabular}

filter was selected to cover both the redshifted $\mathrm{H}_{\alpha}$ and [NII] lines. The underlying continuum was taken through the Gunn $r^{\prime}$ filter. The integration time was $60 \mathrm{~min}(\mathrm{ON}-$ band), split in three shorter exposures to get rid of the cosmic rays, each dithered by 18 arcsec to recover the gap between the 4 chips. The red-continuum frame was exposed $15 \mathrm{~min}$ to avoid saturation on the nucleus of the galaxy. The photometric calibration was obtained exposing the spectrophotometric star Feige 34 on each of the 4 CCD chips. The images were bias subtracted and flatfielded using combinations of exposures on the twiglight sky. After background subtraction and re-alignement, the intensity in the $r^{\prime}$ image was normalized on that in the ON-band one using several field stars. The Net-image was obtained by subtracting the normalized $r^{\prime}$ frame from the ON-band one. The resulting Net-frame is given in Figs. 1 and 2. The individual features are labelled following the nomenclature of G84.

\subsection{Broad-band imaging}

The galaxy UGC 6697 was observed during the science verification period of VLT-UT1 with FORS1 on the night of 11 March, 1999 with seeing of $0.6 \operatorname{arcsec}(I$ band) (see Tables 1 and 3 ). The observations were taken through the $B, V, R, I$ filters with integration times given in Table 1 . Each exposure was split in shorter sub-exposures which were combined using a median filter to eliminate the cosmic rays. The images were bias subtracted and flat-fielded using combinations of archive flat-field exposures. This process did not give satisfactory results at the edge of the frames because of some residual gas contamination in the chip which affected the early measurements with FORS1. However the central $\sim 3 \times 3$ arcmin containing UGC 6697 , did not suffer from such a problem. The combined $R$ band frame is given in Figs. 3 and 4 . The true color map ( $B$, $V, I)$ is shown in Figs. 8 and $9 \mathrm{~b}$.

Archive images of U6697 taken with the HST Wide Field and Planetary Camera (WFPC2) were also inspected. These consist of two $100 \mathrm{~s} I$ band exposures (F814W $\lambda 7940 \AA$ ) and of two 1000 s UV exposures (F300W $\lambda 2910 \AA$ ). These images were combined to produce the true color $U V-I$ frame given in Fig. 9a. An enlargement of the $I$ frame containing the central region around feature A4 is shown in Fig. 10.
Table 2. The spectroscopic observations.

\begin{tabular}{lccccc}
\hline Spectrum Telescope & $\begin{array}{c}\text { Tint } \\
\text { sec }\end{array}$ & $\begin{array}{c}\text { slitPA } \\
\text { deg. }\end{array}$ & $\begin{array}{c}\text { slitwidth } \\
\text { arcsec }\end{array}$ & $\begin{array}{c}\text { Pos.rel.A4 } \\
\text { arcsec }\end{array}$ \\
\hline L 1 M & Loi & $900 \times 3$ & 147 & 2.5 & $2.3 \mathrm{~N}$ \\
L 2 M & Loi & $600 \times 3$ & 147 & 2.5 & 0 \\
L 3 M & Loi & $600 \times 3$ & 147 & 2.5 & $1 \mathrm{~S}$ \\
L 4 M & Loi & $600 \times 3$ & 147 & 2.5 & $3.5 \mathrm{~S}$ \\
L 5 M & Loi & $600 \times 3$ & 57 & 2.5 & $7 \mathrm{~S}$ \\
L 1 m & Loi & $420 \times 3$ & 57 & 2.5 & $6 \mathrm{E}$ \\
L 2 m & Loi & $420 \times 3$ & 57 & 2.5 & $17 \mathrm{~W}$ \\
L 3 m & Loi & $420 \times 3$ & 57 & 2.5 & $23 \mathrm{~W}$ \\
O 1 M & OHP & $600 \times 3$ & 147 & 1.8 & 0 \\
O 2 M & OHP & $600 \times 3$ & 147 & 1.8 & $4.5 \mathrm{~S}$ \\
O 1 m & OHP & $600 \times 3$ & 57 & 1.8 & $6 \mathrm{~W}$ \\
O 2 m & OHP & $600 \times 3$ & 57 & 1.8 & $12 \mathrm{~W}$ \\
\hline
\end{tabular}

\subsection{Long-slit spectroscopy}

We obtained high dispersion long-slit spectra of UGC 6697 with the $1.93 \mathrm{~m}$ telescope of the Observatoire de Haute Provence (OHP), equipped with the CARELEC spectrograph (Lemaitre et al. 1990) coupled with a $512 \times 512$ TK CCD, giving a spatial scale of 1.17 arcsec per pixel. The observations were carried out in the night of March 2, 1998 in approximately 2 arcsec seeing conditions through a slit of $5 \operatorname{arcmin} \times 1.8$ arcsec (see Tables 2, 4). The selected grism gives a spectral resolution of $32 \AA / \mathrm{mm}$ or $0.85 \AA /$ pix and a spectral coverage in the region 6500-6900 $\AA$ containing the redshifted $\mathrm{H}_{\alpha}(\lambda 6562.8 \AA)$, the $[\mathrm{NII}]$ doublet $(\lambda \lambda 6548.1,6583.4 \AA)$ and the $[\mathrm{SII}]$ doublet $(\lambda \lambda 6717.0,6731.3 \AA)$.

Long-slit, intermediate dispersion spectra of UGC 6697 were also obtained in the night of February, 25, 2000 using the imaging spectrograph BFOSC attached to the Cassini $1.5 \mathrm{~m}$ telescope at Loiano (Italy), coupled with a $1340 \times 1300 \mathrm{EEV}$ CCD, giving a spatial scale of 0.58 arcsec per pixel. The selected grism gives a spectral resolution of $81 \AA / \mathrm{mm}$ or $1.62 \AA /$ pix and a spectral coverage in the region $6000-8200 \AA$.

Exposures of a He-Ar lamp secured the wavelength calibration of both sets of observations. This was checked and corrected using the $\lambda 6863.95 \AA$ sky line. The residual systematic wavelength error is within $0.5 \AA$.

In total we took 7 long-slit spectra along PA 147 degrees parallel to the galaxy major axis. These spectra are labelled "M". We took also 5 spectra at PA 57 degrees, i.e. perpendicular to the galaxy major axis. These spectra are labelled "m". Two parallel and two perpendicular spectra were taken at the OHP, the remaining 5 parallel and 3 perpendicular spectra were taken at Loiano. The log book of the long-slit spectroscopic observations is given in Table 2. Both images and spectra were reduced using standard procedures within the IRAF environment.

The exact locations of the slit positions on the galaxy are given in Fig. 5. The $\mathrm{H}_{\alpha}$ frame of Fig. 2 is displayed with shallower contrast after rotating the frame by -57 degrees 


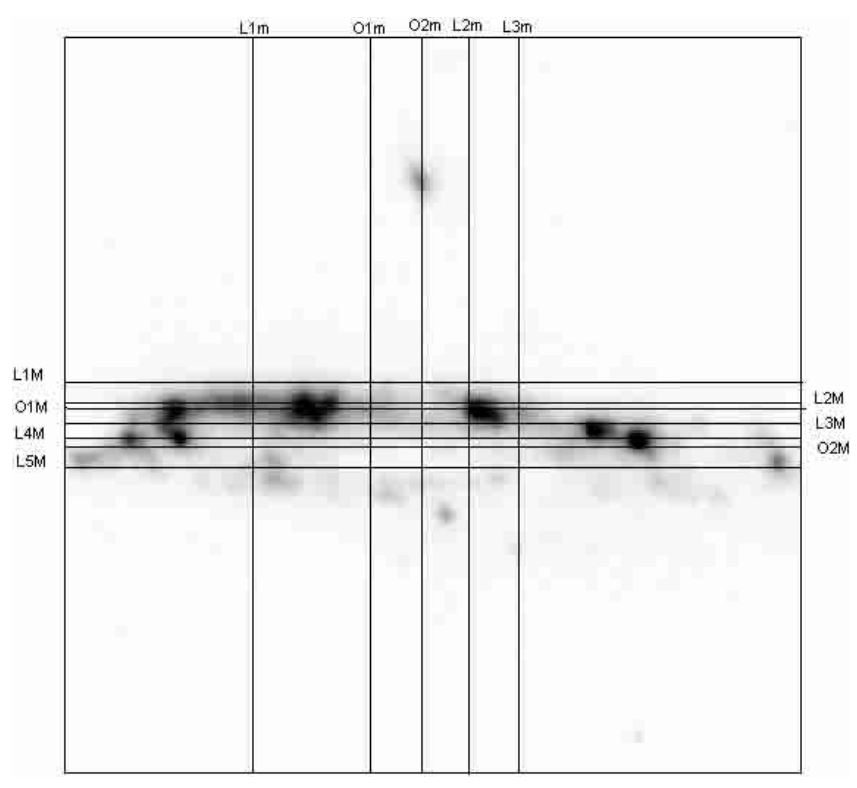

Fig. 5. The net $H_{\alpha}$ frame with shallow contrast, rotated by -57 degrees. The slit positions are superposed. The two outermost vertical lines are drawn with 30 arcsec separation.

to bring the galaxy major axis parallel to the frame $X$ axis, thus parallel to the slit PA 147 (M spectra). The determination of the slit positions was straightforward for the Loiano spectra, since reference images were taken with BFOSC (imaging spectrograph) immediately prior to exposing the spectra. The correspondence of the OHP slit position on the image is less trivial because the pointing with Carelec relies on a TV camera, thus the absolute telescope pointing is not accurately known. However we derived a posteriori the exact location of the long slit spectra on the image as follows: we integrated the intensity distribution along the slit in the spectra. Then we did a similar job on the $\mathrm{H}_{\alpha}$ frame along several rectangular regions of width and length equal to the spectrograph slit, slightly displaced from one another. The two intensity distributions were found in good agreement at the positions indicated in Fig. 5.

The 4 OHP 2-dimensional spectra are given in Fig. 6 (parallel) and Fig. 7 (perpendicular), which immediately shows the peculiarities of the galaxy rotation curve: i.e. the sudden velocity variations. In particular one should notice the high velocity feature in the O1M spectrum which was already pointed out by G84 as a striking peculiarity.

\subsection{The Fabry-Perot observations}

We observed UGC 6697 in April 1999 with a scanning Fabry-Perot placed at the Cassegrain focus of the $1.93 \mathrm{~m}$ telescope of the Observatoire de Haute Provence (GHASP instrument, see http://www-obs.cnrs-mrs.fr/interferometrie/GHASP/ ghasp.html for more information). This (2 h exposure) observation confirmed the high velocity bump (more than $200 \mathrm{~km} \mathrm{~s}^{-1}$ ) observed in slit spectroscopy in the central part of the Galaxy. However, the seeing was rather bad (between 2 and 3 arcsec) and the resulting lack of spatial resolution, added to the high inclination of the object (almost seen edge-on), prevented the Fabry-Perot observations to bring something new compared with the slit spectroscopy. Observations were then repeated in February 2000, with a scanning Fabry-Perot placed at the Cassegrain focus of the $3.6 \mathrm{~m}$ CFHT. The seeing was better than 1 arcsec and the results proved quite interesting because of the high resolution, both spectral $\left(9.5 \mathrm{~km} \mathrm{~s}^{-1}\right.$ sampling) and spatial (0.88 arcsec sampling, with the STIS2 CCD in binning mode). The $\mathrm{H}_{\alpha}$ line of the galaxy was selected through an interference filter centered at $6711 \AA$, with a $12 \AA F W H M$. The Fabry-Perot used for these observations has a free spectral range of $266 \mathrm{~km} \mathrm{~s}^{-1}$ and was scanned through 28 steps. The ambiguity of $k \times 266 \mathrm{~km} \mathrm{~s}^{-1}$ on the velocities was mainly solved owing to the slit spectra measurements. However this problem was especially hard to solve in the central part of the object, where the velocity profile is double peaked, with a peak separation similar to the free spectral range, so that the two line profiles are almost brought in coincidence there (see Sect. 3.4 for a detailed discussion of the velocity profiles analysis). The Fabry-Perot characteristics are given in Table 5.

\section{Results}

\subsection{Morphology}

We shall start the discussion on UGC 6697 by revising the galaxy morphology, taking advantage of the deeper, higher-resolution narrow and broad-band imaging reported in this work, compared with the previously available observational material (G84, G95).

The galaxy, in its total extension of 2.3 arcmin, has a strongly asymmetric light distribution along the major axis: from position A1 to B2 it has a high surface brightness (see Figs. 1-4 and 8), and strong continuum (see Figs. 3, 4 and 12). Further to the NW of B2, the surface brightness decreases significantly up to $\mathrm{D}$, then the patchy trail-like $\mathrm{N}-\mathrm{W}$ extension becomes progressively fainter up to the last visible isophotes.

The bright S-E part of the galaxy (A1 to B2) consists of a thin curved structure connecting a series of relatively bright "knots". The most prominent of these features is A4, which was previously identified with the galaxy nucleus. Immediately to the NW of A4, a pronounced gap in the continuum distribution indicates the presence of strong extinction (see below). More to the NW of the gap the galaxy has a relatively bright continuum (extended from $\mathrm{B} 1$ toward the $\mathrm{NW}$ ), but little or no $\mathrm{H}_{\alpha}$. In other words, the $\mathrm{H}_{\alpha}$ gap (A4 to B2) is twice as extended as the continuum one (A4 to $\mathrm{B} 1$ ). Moreover, the $R$ band frame (see Fig. 4) clearly indicates that feature B2 is displaced to the $\mathrm{N}$ of the bright continuum (B1) and is resolved into several smaller patches. 
Table 3. The imaging instrumental set-up.

\begin{tabular}{lcccc}
\hline Telescope & Date & Camera & CCD & $\begin{array}{c}\text { Pix } \\
\operatorname{arcsec}\end{array}$ \\
\hline INT & 26 Apr. 2000 & WFC & $4 \times 2000 \times 4000$ EEV & 0.33 \\
VLT-UT1 & 11 Mar. 1999 & FORS1 & $2048 \times 2048$ TK & 0.20 \\
\hline
\end{tabular}

Table 4. The spectrograph characteristics.

\begin{tabular}{|c|c|c|c|c|c|c|c|}
\hline Telescope & Date & Spectrograph & $\begin{array}{c}\text { Dispersion } \\
\AA / \mathrm{mm}\end{array}$ & $\begin{array}{c}\text { Dispersion } \\
\AA / \text { pix }\end{array}$ & CCD & $\begin{array}{l}\text { pix } \\
\mu \mathrm{m}\end{array}$ & $\begin{array}{l}\text { Spat. Scale } \\
\text { "/pix }\end{array}$ \\
\hline OHP & 2 Mar. 1998 & CARELEC & 32 & 0.85 & $512 \times 512$ TEK & 27 & 1.17 \\
\hline Loiano & 25 Feb. 2000 & BFOSC & 81 & 1.62 & $1340 \times 1300 \mathrm{EEV}$ & 20 & 0.58 \\
\hline
\end{tabular}

Table 5. The Fabry-Perot observations characteristics.

\begin{tabular}{lcccccccc}
\hline Telescope & Date & Detector & $\begin{array}{c}\text { Filter } \\
\AA\end{array}$ & $\begin{array}{c}\text { Free spec. range } \\
\mathrm{km} \mathrm{s}^{-1}\end{array}$ & $\begin{array}{c}\text { Detector } \\
\begin{array}{c}\text { Pixel scale } \\
\mu \mathrm{m}\end{array}\end{array}$ & $\begin{array}{c}\text { Tint } \\
\text { "/pix }\end{array}$ & $\mathrm{s}$ \\
\hline OHP & 13 Apr. 1999 & IPCS & $6723(50)$ & 376 & $256 \times 256$ & 37 & 0.96 & 7500 \\
CFHT & 26 Feb. 2000 & STIS2 & $6711(12)$ & 266 & $2048 \times 2048$ & 21 & 0.88 & 3360 \\
\hline
\end{tabular}

Also perpendicular to the major axis the galaxy is very asymmetric: the bright structure described above lies on the NE edge of the object, while the SW part (A1, A2, $\mathrm{R} 1, \mathrm{R} 2, \mathrm{R} 3, \mathrm{R} 4$ ) is much fainter and patchy, the two being separated by a gap with almost no $\mathrm{H}_{\alpha}$ emission.

The color distribution (see Fig. 8), going from SE to NW indicates a very blue color $(B-V \sim 0.3)$ in the complex A1, followed by a very red A4 $(B-V \sim 1.0)$ and its surrounding region, including $\mathrm{B} 1$. West of B1, i.e. from $\mathrm{B} 2$ to $\mathrm{D}$, the patchy structure is very blue $(B-V \sim 0.2)$. Summarizing, the central region of the galaxy (corresponding with the region with little $\mathrm{H}_{\alpha}$ emission) is red, with several patchy, dusty regions and a relatively high extinction as measured by the Balmer decrement, as opposed to the periphery where the color is blue, suggesting a strong absorption in the central part. Whereas the continuum morphology is consistent with that of a disk galaxy seen almost edge-on, the $\mathrm{H}_{\alpha}$ and the color frames indicate a more complex morphology.

The central region containing A4 deserves special attention. As stated above A4, being the reddest and brightest spot (the brightest feature in the $H$ band image reported in G95), was tentatively identified with the galaxy nucleus. Seen at the high spatial resolution in both the VLT and HST (Figs. 8, 9 and 10) images, A4 appears resolved into two sub-features with distinctly different color: a bluer, fainter one to the north and a redder, brighter southern spot. Although it cannot be excluded that the two features are produced by dust-obscuration (a subarcsecond seeing NIR image would be necessary to shed light on this point), we propose to identify the northern spot as the nucleus of the main galaxy and the southern one with the nucleus of the second galaxy discussed in Sect. 4 .

\subsection{The long-slit velocity measurements}

Velocity plots of UGC 6697 were extracted from each of the spectra by measuring the wavelength of the $\mathrm{H}_{\alpha}$ line in each pixel along the slits. The Loiano spectra, which are noisier due to the smaller telescope aperture, were smoothed with a Gaussian filter of 1 pixel kernel. The 7 plots parallel to the galaxy major axis are given in Fig. 11 together with the 5 plots perpendicular to the galaxy major axis (right panels). In each diagram the recessional velocity is plotted as a function of position along the slit (the spatial axis runs from SE (left) to NW (right) for the major-axis spectra; from NE (left) to SW (right) for the minor-axis spectra). The vertical solid lines mark the positions of the bright features (as labelled in Fig. 1), whereas the dotted vertical lines mark the position along which the perpendicular spectra were taken. All of the four spectra taken at OHP show regions with multiple velocity components (cf. Figs. 6 and 7). This is not the case in the Loiano spectra, due to their shallower intensity combined with lower dispersion. Broadly speaking, the parallel velocity plots are consistent with a steep linear velocity increase by about $400 \mathrm{~km} \mathrm{~s}^{-1}$ from A1 to approximately B2. This is consistent with the rotation curve of a rigid body, or of a disk seen at large inclination. From B2 to D the rotation curve is significantly flatter. Furthermore, in the intermediate region A4-B2, all spectra, except the southernmost one (L5M), show a high velocity "bump" with a sudden velocity increase by approximately $200 \mathrm{~km} \mathrm{~s}^{-1}$.

The perpendicular spectra L1m (taken to the SW of A4) shows almost no velocity gradient across the galaxy. All other perpendicular spectra begin with a low velocity component (corresponding to the Northern edge of the galaxy), a rapid increase in velocity by approximately $200 \mathrm{~km} \mathrm{~s}^{-1}$, followed by a smooth velocity decrease. 

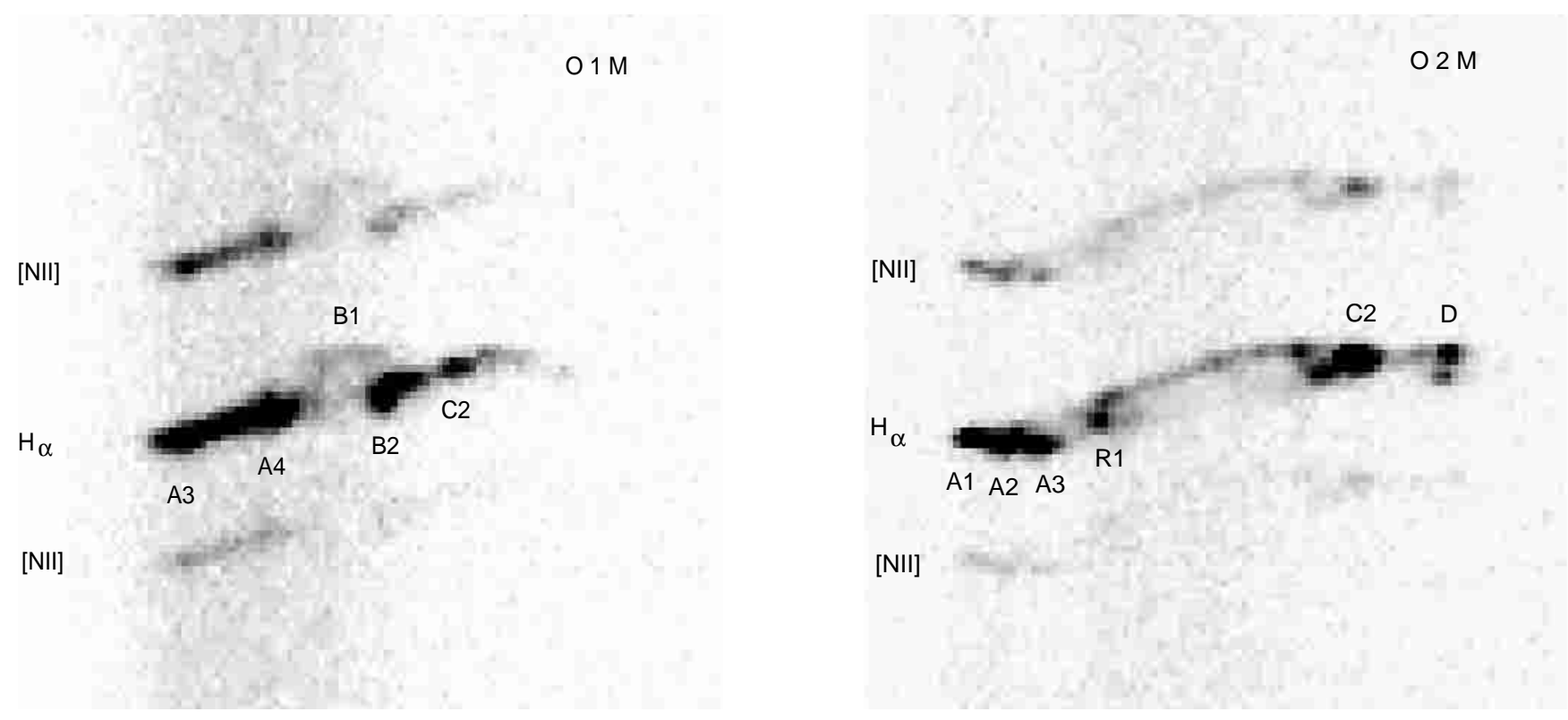

Fig. 6. The two OHP parallel spectra taken at position O1M (left), O2M (right). The wavelength increases from bottom to top. $\mathrm{E}$ is to the left, $\mathrm{W}$ to the right.
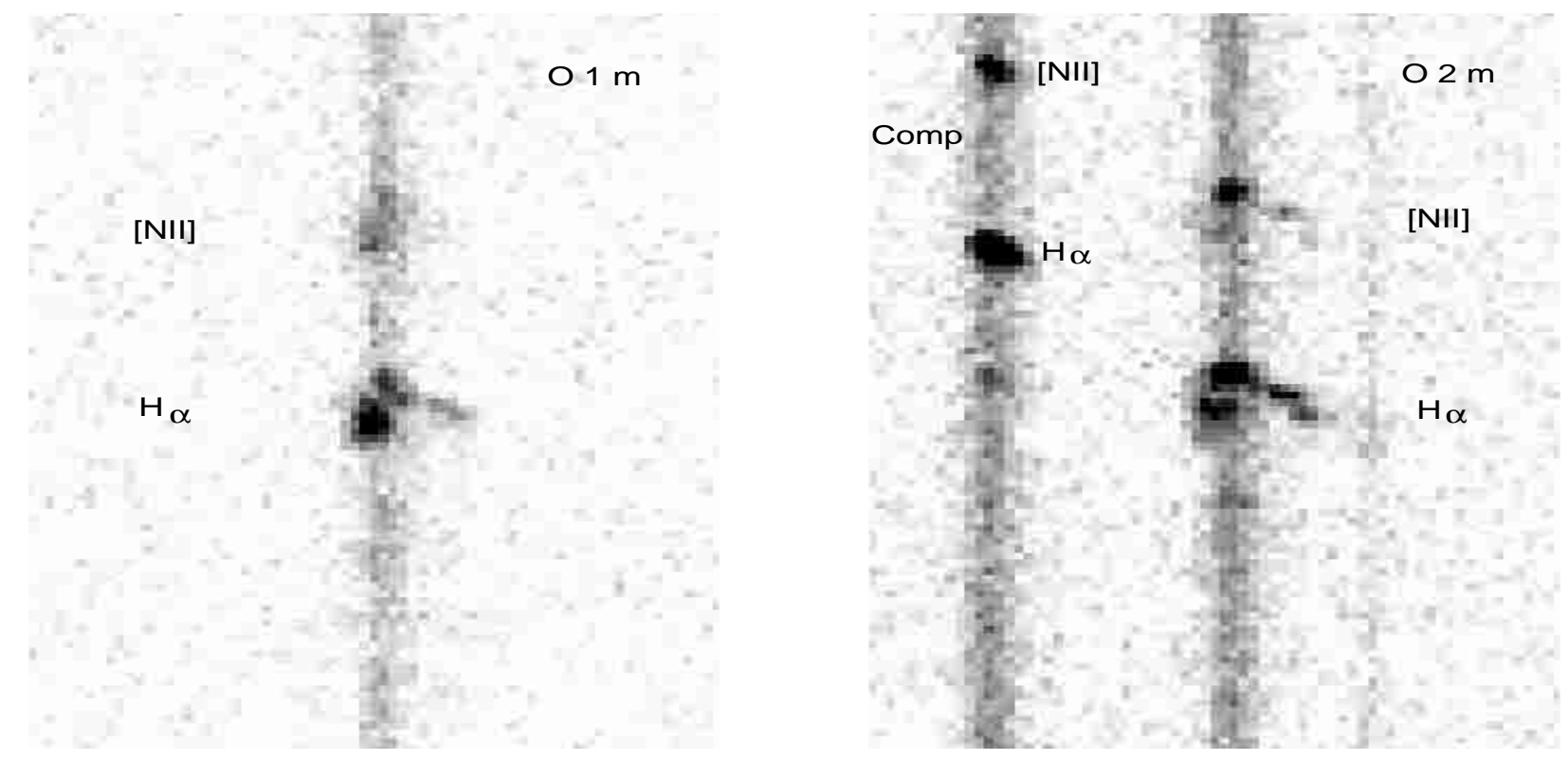

Fig. 7. The two OHP perpendicular spectra taken at position $\mathrm{O} 1 \mathrm{~m}$ (left), O2m (right). The $\mathrm{H}_{\alpha}$ line from the companion galaxy is visible. The wavelength increases from bottom to top. $\mathrm{N}$ is to the left, $\mathrm{S}$ to the right.

The spectrum L3m shows, in addition to these features, a very low velocity component $\left(\sim 6700 \mathrm{~km} \mathrm{~s}^{-1}\right)$ corresponding with feature R3. This low value suggests that R3 is an isolated blob of ionized gas, separated from the main body of the galaxy. Depending on the geometry of the object, it could be as well a cloud detaching from the galaxy or falling onto it.

\subsection{Metallicity and extinction}

To study the metal content and the internal extinction in the various features, we took also a low dispersion spectrum along the major axis of the galaxy (see Fig. 12). The low dispersion spectra extracted for each single region are shown in Fig. 13. Table 6 lists the line parameters of the individual feature (Col. 1) as follows: the extinction (at $\mathrm{H}_{\alpha}$ in mag) is given in Col. 2, the underlying Balmer absorption $(\AA)$ (Col. 3); the dereddened [OIII] and [NII] line strenght normalized to $\mathrm{H}_{\alpha}$ (Cols. 4 and 5) used to derive the oxygen abundances given in Col. 6. All regions, except B1, show strong Balmer emission and [OIII] emission lines typical of actively star forming HII regions. The extinction, as derived from the Balmer decrement following Boselli et al. (2001), appears negligible at all positions, except at B1 and B2, in agreement with the color map. 


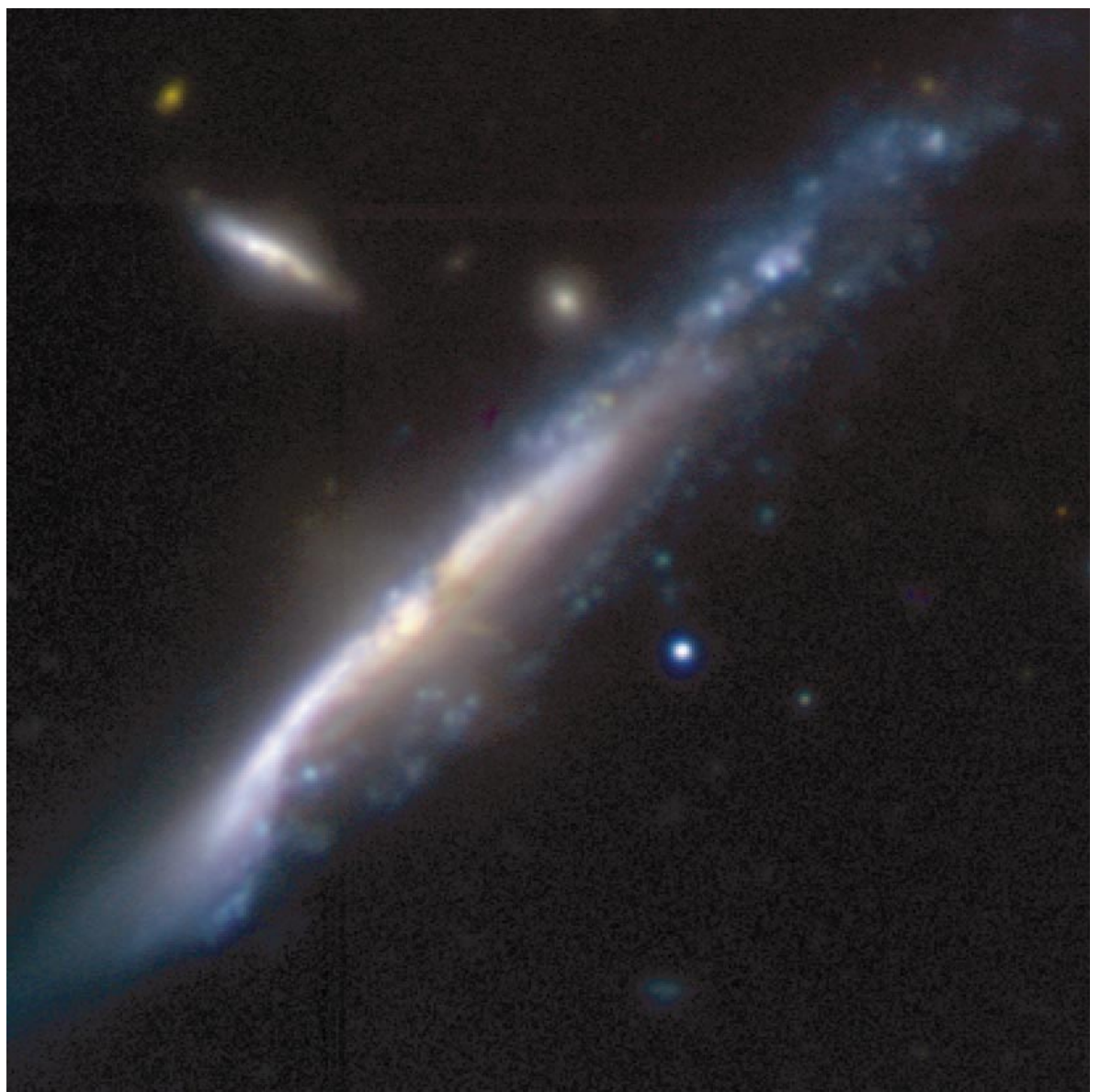

Fig. 8. The true color VLT image $(B, R, I)$. The logarithmic color scale is such that the bluest regions have $B-V \sim 0.2$ and the reddest $B-V \sim 1.0$.
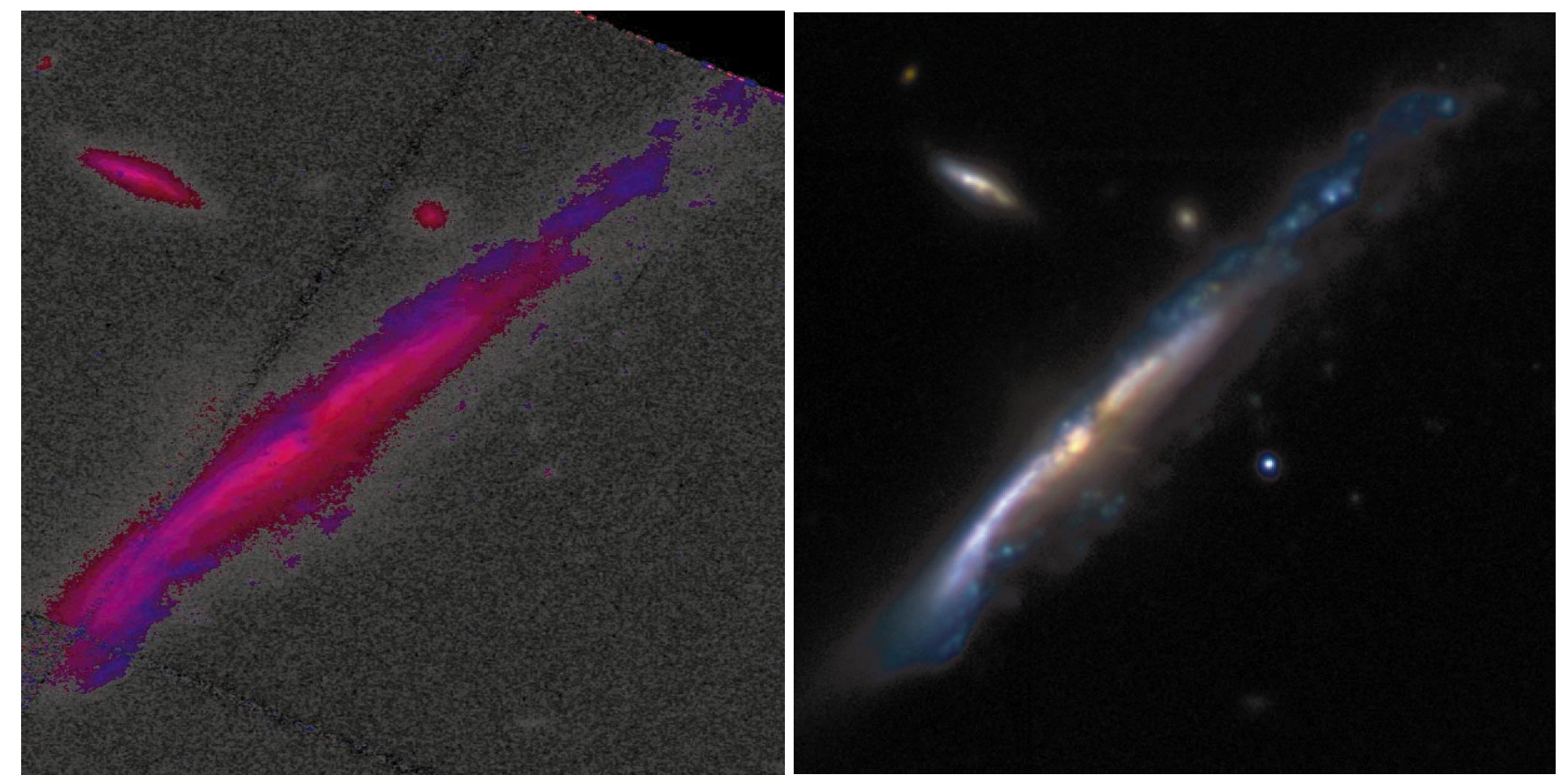

Fig. 9. The true color HST image $(U V, I)$ (left) and the true color VLT image $(B, R, I)$ (right) (same as Fig. 8 with linear intensity scale that enhances the bright features). 
Table 6. The line parameters of the individual features.

\begin{tabular}{|c|c|c|c|c|c|}
\hline Feature & $\begin{array}{l}A_{\mathrm{H} \alpha} \\
\mathrm{mag}\end{array}$ & $\begin{array}{c}E W(\mathrm{H} \beta) \\
\AA\end{array}$ & {$[\mathrm{OIII}] / \mathrm{H} \alpha$} & {$[\mathrm{NII}] / \mathrm{H} \alpha$} & $\begin{array}{c}Z \\
12+\log (\mathrm{O} / \mathrm{H})\end{array}$ \\
\hline $\mathrm{A} 1$ & 0.0 & -7.87 & 0.25 & 0.25 & 8.80 \\
\hline A4 & 0.2 & - & 0.62 & 0.30 & 8.68 \\
\hline B1 & 1.1 & -8.47 & $<0.07$ & 0.36 & $>9.10$ \\
\hline B2 & 1.7 & - & 0.57 & 0.34 & 8.71 \\
\hline $\mathrm{C} 12$ & 0.0 & - & 0.99 & 0.18 & 8.51 \\
\hline $\mathrm{D}$ & 0.0 & - & 1.09 & 0.18 & 8.50 \\
\hline
\end{tabular}

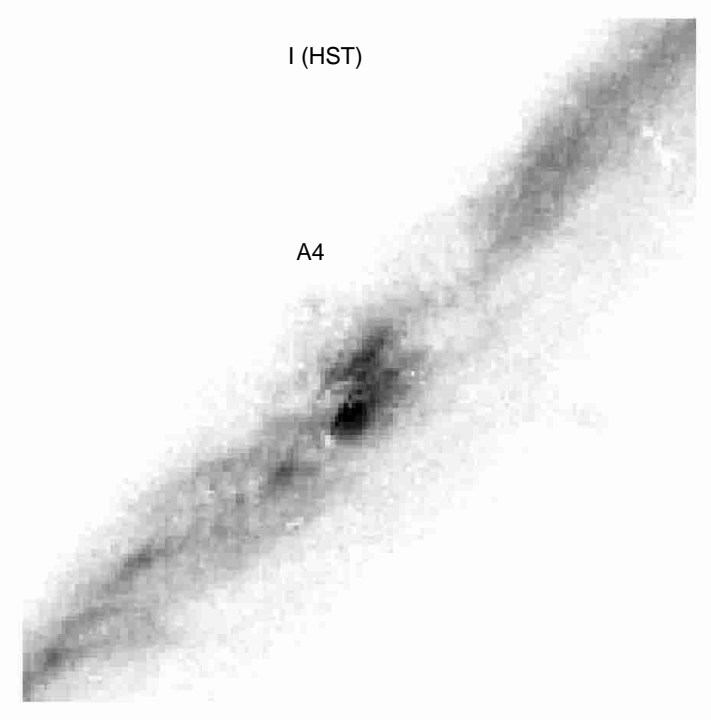

Fig. 10. A $(\sim 20 \times 20$ arcsec $)$ enlargement of the central region of the HST $I$ band image showing that feature A4 is resolved into two components.

The underlying Balmer absorption is negligeable except in A1 and B1. The spectra of regions B1, and to a lesser extent A1 (and A4), show other conspicuous absorption lines: $\mathrm{Mg} \mathrm{I}(5175 \AA)$, plus several iron absorption features at $\lambda \lambda 5270,5406 \AA$. The Na ID absorption can be seen also, very intense at B1.

Metallicities are estimated from the $[\mathrm{OIII}] /[\mathrm{NII}]$ corrected line ratio, using the empirical calibration of Edmunds \& Pagel (1984). All regions except B1 show consistent oxygen abundances (8.5-8.8), slightly lower than solar. In region $\mathrm{B} 1$, the upper limit to the $[\mathrm{OIII}] /[\mathrm{NII}]$ line ratio translates into a lower limit to the metallicity of 9.1, significantly higher than in all other regions.

Altogether it appears that all features except B1 are actively star forming regions. B1 seems to have a different nature: it has almost suppressed the current star formation, while it shows evidence of post-starburst activity, it has significantly lower excitation parameter and higher metallicity (Dopita et al. 2000).

\subsection{The 2-D velocity field}

Figure 14 shows some examples of the various types of the $\mathrm{H}_{\alpha}$ profiles observed with the scanning Fabry-Perot. The central map used to identify the place where each profile is observed is the crude $\mathrm{H}_{\alpha}$ map obtained from the scanning sequence, when adding all the interferograms (28 interference patterns obtained through the different scanning steps). It shows the $\mathrm{H}_{\alpha}$ intensity effectively observed through our combination of interferential filter and Fabry-Perot interferometer. The eleven selected profiles are commented hereafter (counterclockwise from top left).

N.B. The wavelength origin is the same for all profiles. However, when looking at these profiles, one must keep in mind that the free spectral range is only $266 \mathrm{~km} \mathrm{~s}^{-1}$, so that the $\mathrm{H}_{\alpha}$ emission line often appears with one wing seemingly cut and continued on the other side of the diagram (details are given in the text hereafter for each profile to clarify this problem). Also, when there are double profiles, due to superimposed emission areas on the line of sight, the two components of the profile may be indeed separated by one free spectral range, so that the velocity scale on the $X$ axis of the diagram (going from $6956 \mathrm{~km} \mathrm{~s}^{-1}$ up to $7222 \mathrm{~km} \mathrm{~s}^{-1}$ ) has to be shifted from + or $-266 \mathrm{~km} \mathrm{~s}^{-1}$ in some cases (one had to refer to the slit spectra to know the right shift to be applied, especially when strong velocity jumps prevented to find the right value by continuity from one pixel to the next). This explains the changes in the velocity scales plotted on the frames of the profiles in Fig. 14, but also the necessity to use simultaneously two different scales on the same frame in some cases, as can be seen on top and bottom of profiles 1,5 and 11 .

Finally, to make reading easier, we normalized the observed profiles. Plotting the true intensity in all the diagrams would have led to flatten the faintest profiles, since there is a factor 10 between the brightest knot (C1, profile 9 ) and the faintest feature displayed here (NW side of R2, profile 7).

- Profile 1: Double profile observed in B2. The brighter component, at about $7020 \mathrm{~km} \mathrm{~s}^{-1}$, belongs to what is called hereafter the second galaxy, meanwhile the main galaxy corresponds here to the other component, at about $6830 \mathrm{~km} \mathrm{~s}^{-1}$.

- Profile 2: Double profile observed in B1. The two components have approximatively the same intensity, one is 


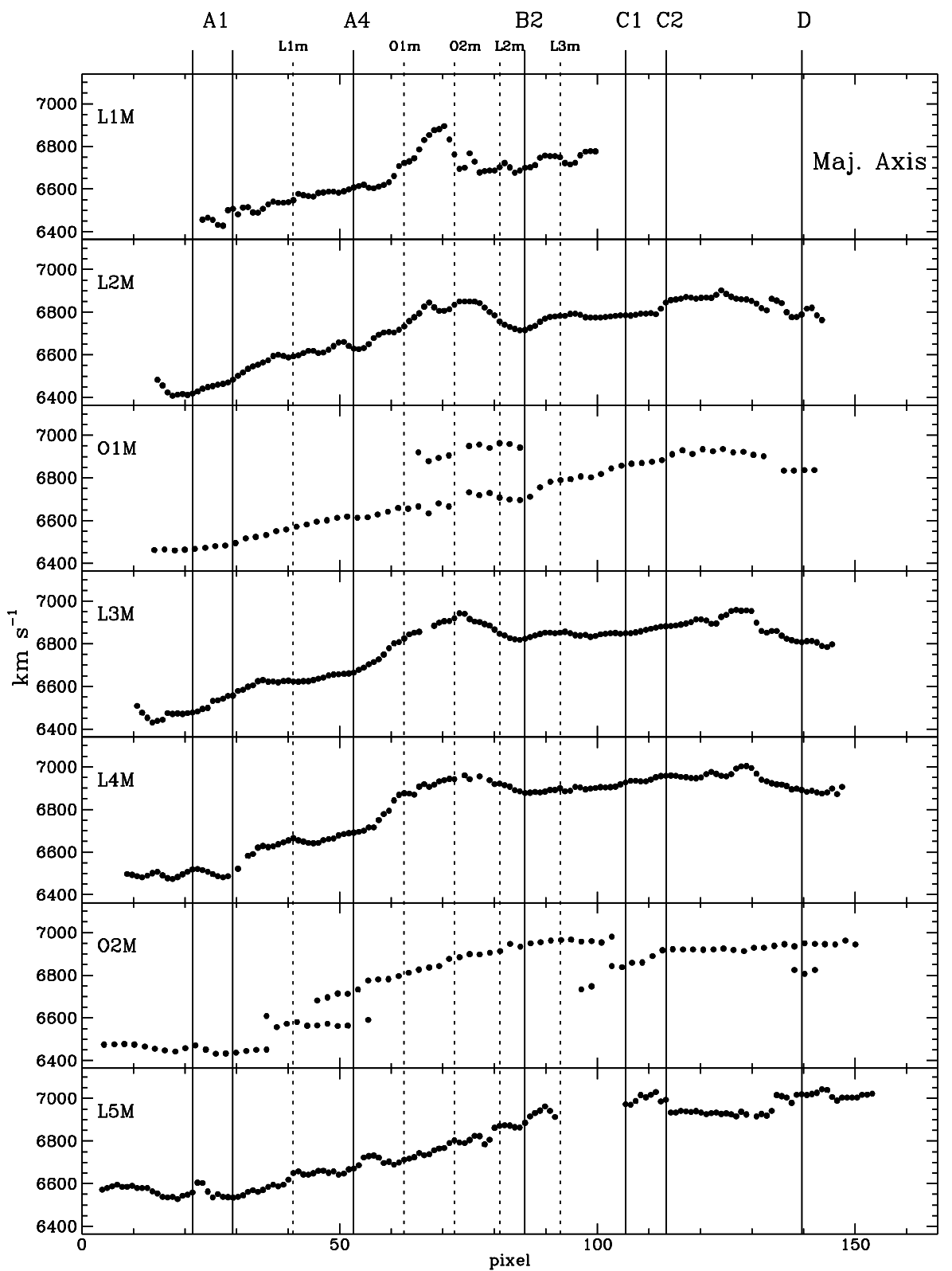

min. axis

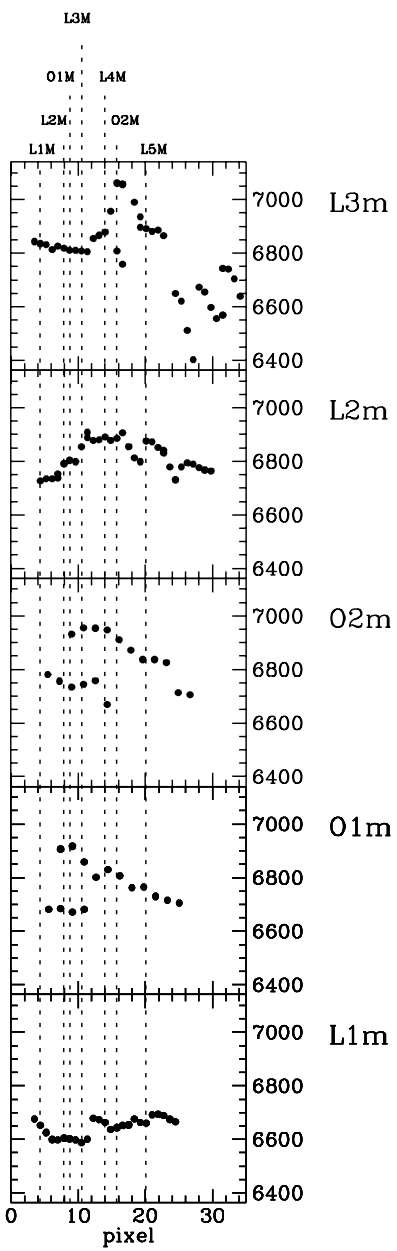

Fig. 11. The 7 parallel (left) and the 5 perpendicular (right) rotation curves obtained from long-slit spectroscopy. The spatial scale is 0.58 arcsec/pixel.

found at about $6710 \mathrm{~km} \mathrm{~s}^{-1}$ (main galaxy) and the other one at about $6880 \mathrm{~km} \mathrm{~s}^{-1}$ (second galaxy). Note that the component at $6710 \mathrm{~km} \mathrm{~s}^{-1}$ is almost centered on the left side of the diagram, so that its left wing appears on the right side of the diagram).

- Profile 3: Simple profile observed in A4. The $\mathrm{H}_{\alpha}$ profile observed in the nucleus area of the main galaxy is rather large and found at about $6700 \mathrm{~km} \mathrm{~s}^{-1}$. As in case of profile 2 , the right wing of the emission line is seen on the left side of the diagram and its left wing on the right side.

- Profile 4: Simple profile observed in A3. The profile is centered at about $6530 \mathrm{~km} \mathrm{~s}^{-1}$ and corresponds to the main galaxy.
- Profile 5: Double profile observed to the south of A4. The component at about $6740 \mathrm{~km} \mathrm{~s}^{-1}$ corresponds to the second galaxy and the component at about $6630 \mathrm{~km} \mathrm{~s}^{-1}$ corresponds to the main galaxy.

- Profile 6: Simple profile observed in R1. This profile, at about $6700 \mathrm{~km} \mathrm{~s}^{-1}$, corresponds to the second galaxy, it is rather large and asymetric, probably because it is contaminated by the emission of the main galaxy. Here again the left wing of the emission line is seen on the right side of the diagram and the right wing on the left side.

- Profile 7: Double profile observed on the NW side of R2. The component at about $6760 \mathrm{~km} \mathrm{~s}^{-1}$ seems to correspond to the main galaxy (but could be as well an isolated blob since it is detached from it) and the component at about $6860 \mathrm{~km} \mathrm{~s}^{-1}$ corresponds to the second 


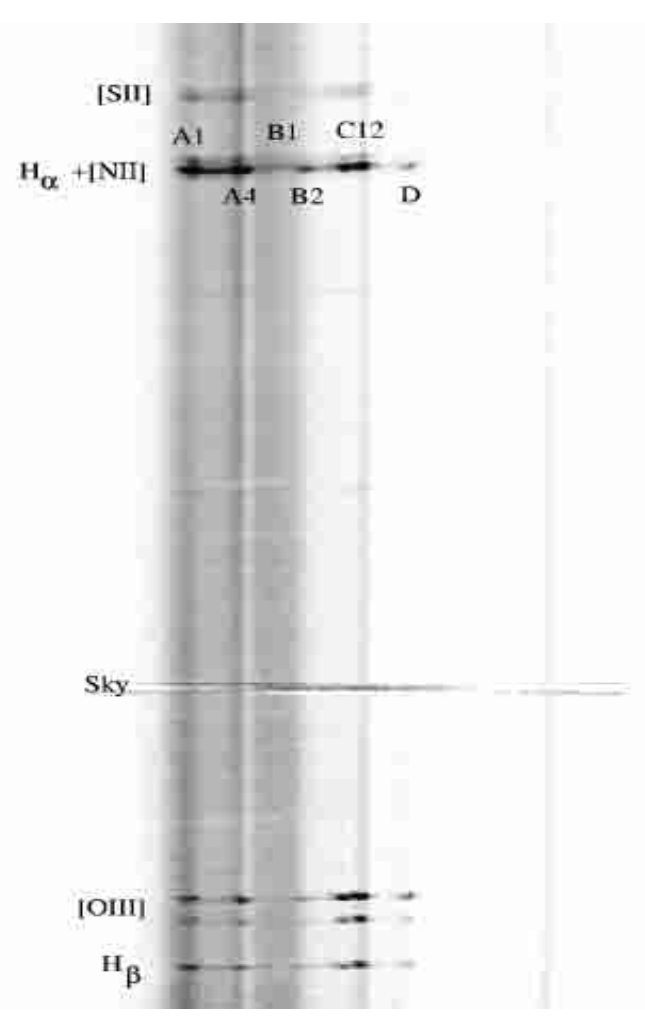

Fig. 12. The low dispersion spectrum taken along the galaxy major axis. The individual features are labelled.
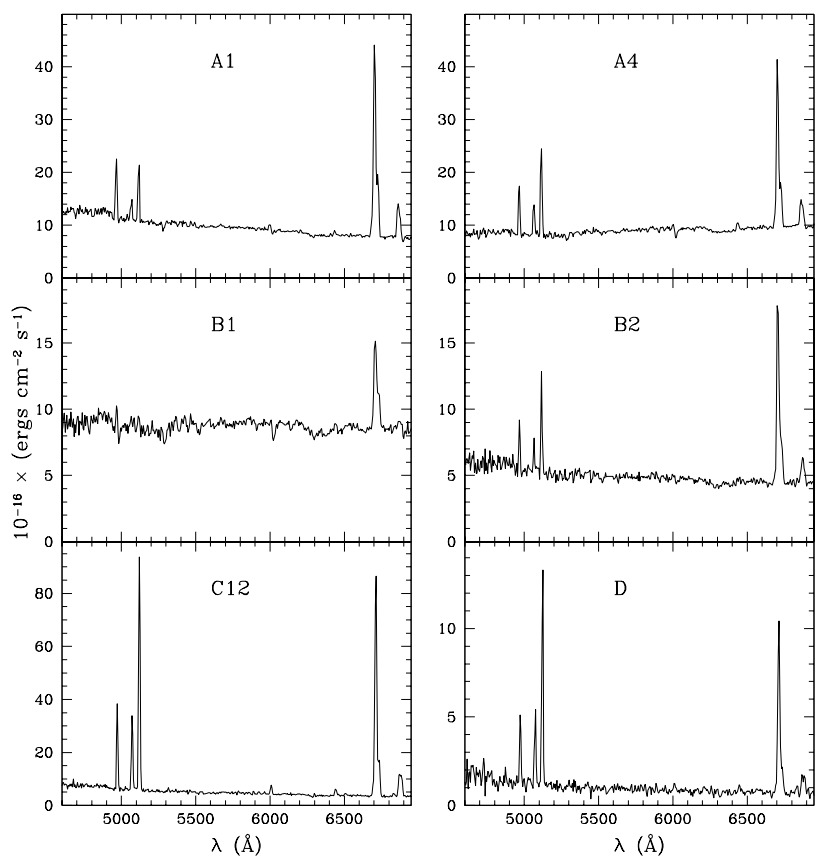

Fig. 13. The 1-dimensional low dispersion spectra of the individual features.

galaxy. Note that the profile observed in the central part of R2 (not shown here) also shows the two components but no so clearly since the one at 6760 clearly dominates. The result is an asymetric large profile.

- Profile 8: Simple profile observed in the bright knot lying about 7 arcsec NW of R2. This profile, at bout

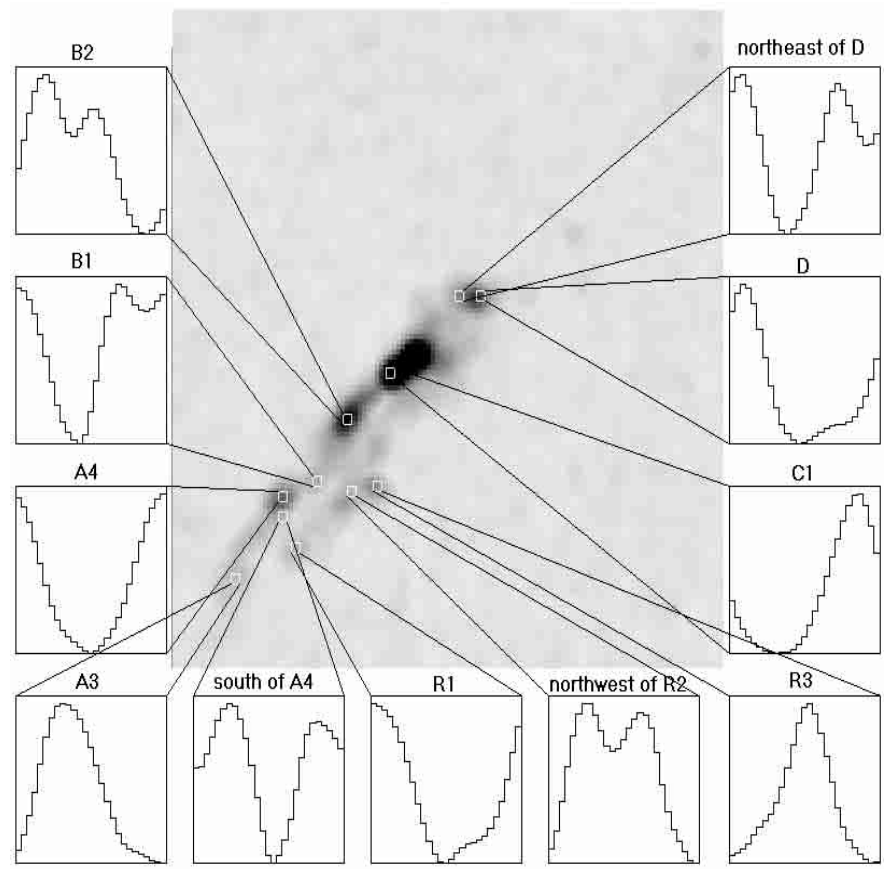

Fig. 14. Examples of the $\mathrm{H}_{\alpha}$ profiles observed in UGC 6697 with the scanning Fabry-Perot.

$6830 \mathrm{~km} \mathrm{~s}^{-1}$, is rather thin. It corresponds to the second galaxy.

- Profile 9: Simple profile observed in C1. By far the brigthest emission region, it exhibits a thin profile at about $6910 \mathrm{~km} \mathrm{~s}^{-1}$, corresponding to the main galaxy. Note that the profile observed in $\mathrm{C} 2$, not shown here, is quite similar but centered at $6970 \mathrm{~km} \mathrm{~s}^{-1}$.

- Profile 10: Simple profile observed in D. This profile, at about $6980 \mathrm{~km} \mathrm{~s}^{-1}$, is peculiarly thin (note that the left wing of the line is on the right side of the diagram) and corresponds to the main galaxy.

- Profile 11: Double profile observed to the NE of D. The main component, at $6980 \mathrm{~km} \mathrm{~s}^{-1}$, is seen again, but now with a component at $6880 \mathrm{~km} \mathrm{~s}^{-1}$ of same intensity. When going further out of the galaxy, toward the NE, the main component disappears and the second one dominates, revealing an isolated blob of ionized gas. Other isolated blobs are found all around the main galaxy as discussed in the text below.

Figures $15 \mathrm{a}$ and $\mathrm{b}$ show the color coded $2-\mathrm{D}$ velocity field obtained from the Fabry-Perot data with contours of the $R$ and the net $\mathrm{H}_{\alpha}$ emission superposed. At the positions where the $\mathrm{H}_{\alpha}$ profile is split into double velocity components, we selected the strongest of the two for this plot. However, when extracting separately the velocity fields for the main galaxy and the second galaxy shown in Fig. 18 we selected independently each component (in case of double profile) and followed the velocity trend by continuity, from one pixel to the neighbouring one. Simulating slit spectra on the 2D Fabry-Perot data enables us to reproduce fairly well the velocity plots of Fig. 11, showing that we have solved in a satisfactorily manner the problem of our narrow free spectral range. 

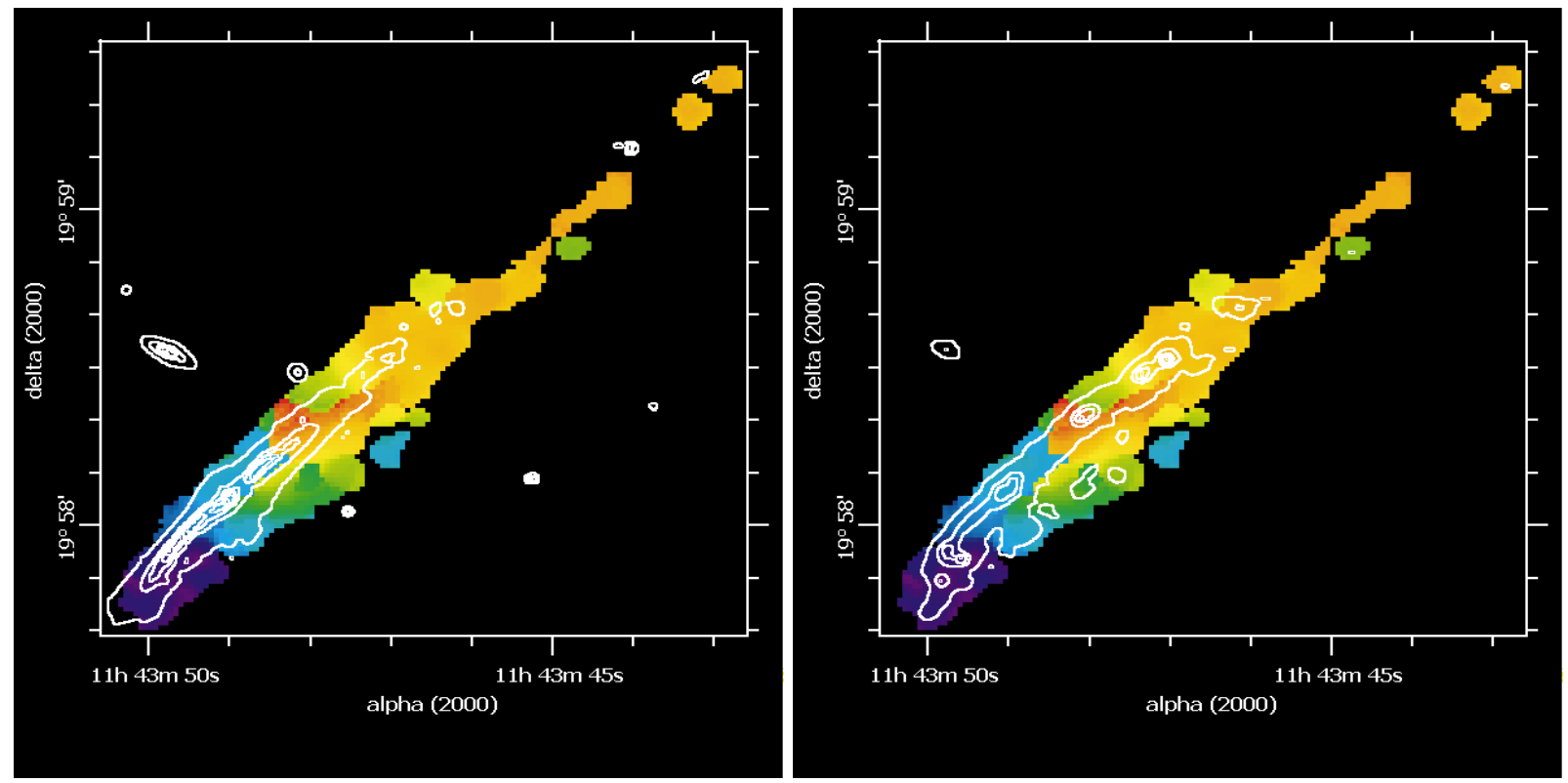

Fig. 15. The color-coded 2-D Fabry-Perot velocity field. Superposed are the contours of the $R$ band image (left) and of the $H_{\alpha}$ net image (right). Velocities are color-coded as in Fig. 16.
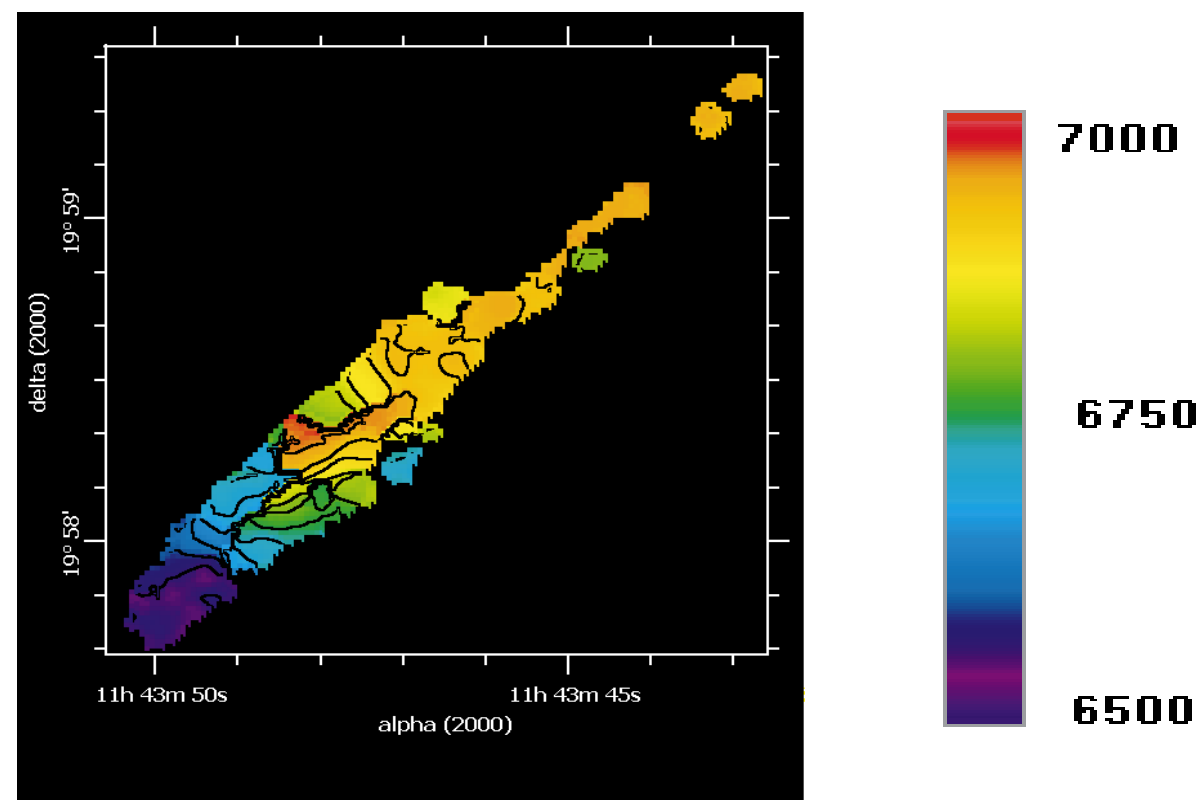

Fig. 16. The color-coded 2-D Fabry-Perot velocity field with superposed iso-velocity contours.

The split of about $200 \mathrm{~km} \mathrm{~s}^{-1}$ seen on the slit spectra (Sect. 3.2) appears mainly in two places in the galaxy, between knots A4 and B2 (including B1). It can be seen on profiles 1 and 2 of Fig. 14. There are other places where a clear split can be seen, although not so large. Examples can be seen in Fig. 14, with profile 5 (south of A4) and profile 7 (northeast of $\mathrm{R} 2$ ) for which the split is around $100 \mathrm{~km} \mathrm{~s}^{-1}$. Except for the case of isolated blobs, mentioned at the end of this section, most of the spliting cases are found in the same area, between A4, B1, B2, $\mathrm{R} 1$ and R2, strongly suggesting that there are two objects seen on the same line of sight in this area. Once again we conclude that the overall appearance of the velocity field along the major axis is that of a rigid body (from A1 $\left(6500 \mathrm{~km} \mathrm{~s}^{-1}\right)$ to $\left.\mathrm{B} 2\left(7000 \mathrm{~km} \mathrm{~s}^{-1}\right)\right)$, flattening out in the NW tail (where the velocity remains approximately constant around $7000 \mathrm{~km} \mathrm{~s}^{-1}$ ). Far in the tail we detect an individual spot with significantly lower velocity, around $6800 \mathrm{~km} \mathrm{~s}^{-1}$ (light green spot in Fig. 15). This small spot is perhaps disconnected from the main object and its velocity could be as well different by $k \times 266 \mathrm{~km} \mathrm{~s}^{-1}$ since no long-slit spectrum enables to check that. Four other isolated spots have abnormally low velocities, compared with the main galaxy :- R3, found around $6710 \mathrm{~km} \mathrm{~s}^{-1}$ (deep green in Fig. 15), had its velocity checked owing to spectrum L3m. - A small spot, a few arcsec NW of 

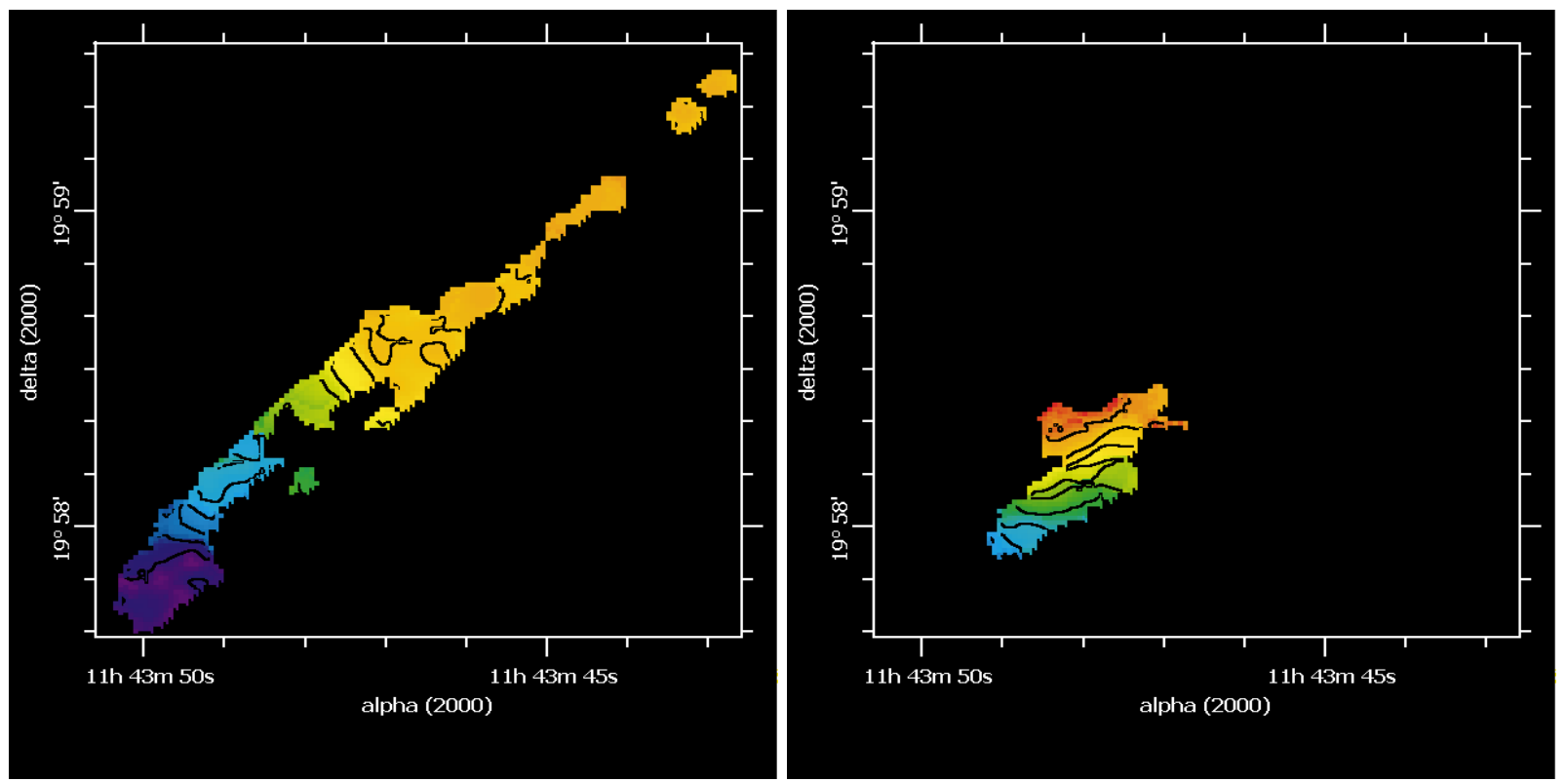

Fig. 17. The color-coded 2-D Fabry-Perot velocity fields of the main and second galaxy with superposed iso-velocity contours.
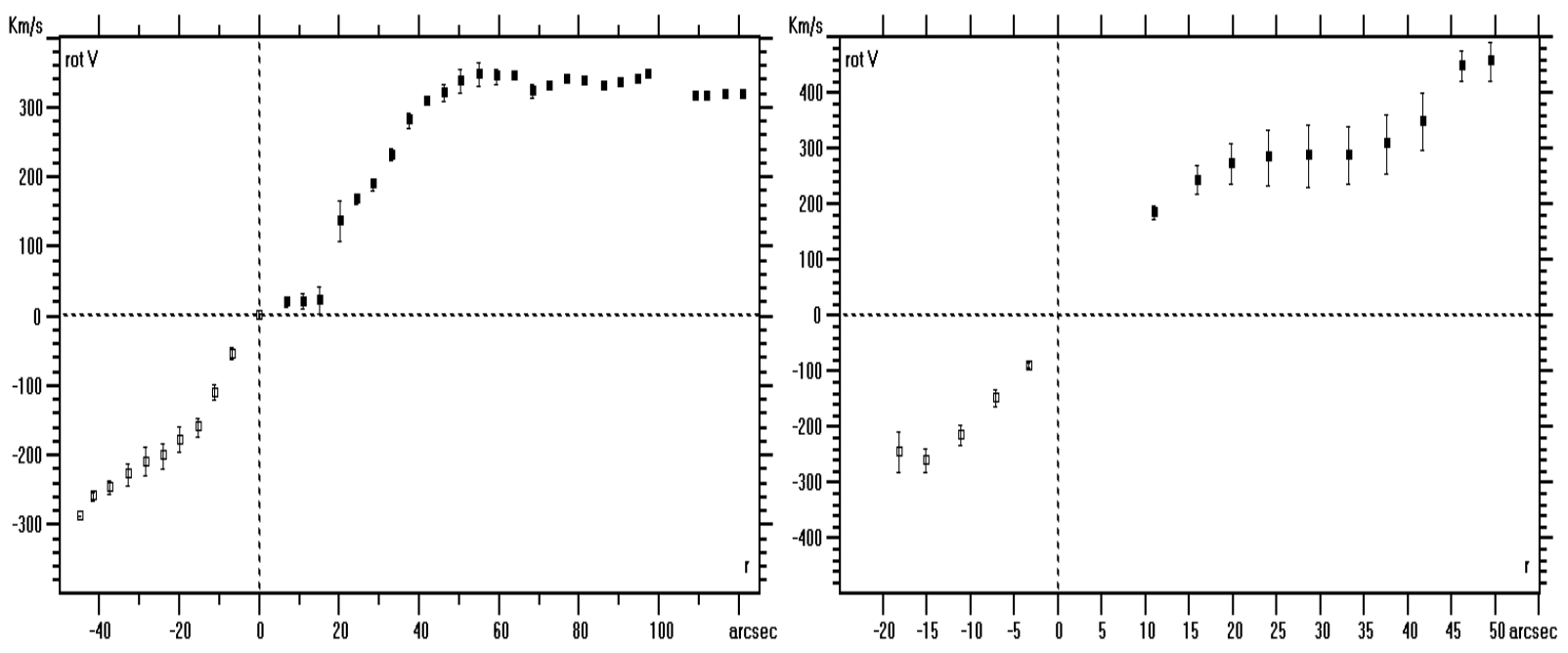

Fig. 18. Rotation curves of the main (left) and second galaxy (right) respectively.

R3, touches the main body of UGC 6697 and appears at about $6830 \mathrm{~km} \mathrm{~s}^{-1}$ (light green in Fig. 15). - The spot NE of knot D, at $6800 \mathrm{~km} \mathrm{~s}^{-1}$ (light green in Fig. 15), had its velocity checked owing to spectrum O2m. Its emission line can be seen on profile 11 of Fig. 14. - R2 is at about $6760 \mathrm{~km} \mathrm{~s}^{-1}$ (see profile 7 in Fig. 14 and isolated light green spot in Fig. 15) and is maybe an isolated blob since it appears separated from the main body of UGC 6697 and there is no clear velocity continuity. However it cannot be related to the second galaxy seen on the same of sight since its velocity profile is clearly separated. Indeed its velocity is not far from what would be expected by extrapolating toward the south the velocities observed in the main body of UGC 6697, so that the knot R2 may be considered as part of the main galaxy meanwhile all the other cases discussed hereabove are probably isolated blobs of ionized gas. These spots are maybe merely satellites of UGC 6697, unless they are the result of the interaction between the two main galaxies, which could explain as well their peculiar velocities.

\section{Discussion}

The most striking peculiarity of the velocity field of UGC 6697 is the sudden velocity jump, more than $200 \mathrm{~km} \mathrm{~s}^{-1}$, observed in the central part in long-slit spectra and confirmed by Fabry-Perot observations.

Because of the nearby companion, at about 30 arcsec NE from UGC 6697, one may think of a Cartwheel-like galaxy seen edge on. However in this case the expanding 
ring would have an expansion velocity about 5 times larger (Amram et al. 1998) which implies an enormous energy amount, 25 times larger, if the mass is the same (in fact the problem is even worse since the velocities measured indicate a larger mass for UGC 6697 compared with the Cartwheel galaxy). Another problem is that the collision time may be estimated to about $165 \times 10^{6}$ years (assuming a constant velocity expansion and a distance of $90 \mathrm{Mpc}$ ). If the companion galaxy has crossed the plane of UGC 6697 perpendicularly, then its tangential velocity, deduced from its present location, must be less than $100 \mathrm{~km} \mathrm{~s}^{-1}$. This is clearly insufficient for such a small companion to have generated the necessary energy to make the main galaxy expand. Also the companion shows no sign of distortion that would have affected it. Finally, the difference in radial velocity between the two (about $800 \mathrm{~km} \mathrm{~s}^{-1}$ ) shows that the companion could not have crossed the main galaxy perpendicularly to its disk but, if it did, sideways.

Another explanation for the double profiles observed could be a strong warp of the highly inclined disk of UGC 6697 due perhaps to the interaction with the small companion visible at $\alpha=11^{\mathrm{h}} 43^{\mathrm{m}} 48.2^{\mathrm{s}} ; \delta=19^{\mathrm{o}} 58^{\prime} 30^{\prime \prime}$. Such a warp is probably real, as can be seen for instance on the SE side in Fig. 8. It can bring different parts of the same rotating disk to appear on the same line of sight with different velocities, thus producing double profiles. However, due to the small mass of the companion (for which unfortunately we have no redshift information), one can hardly explain large velocity separations such as those observed $\left(\sim 200 \mathrm{~km} \mathrm{~s}^{-1}\right)$, in particular close to the minor axis where the velocity should remain approximatively the same. We conclude that the double profiles of Fig. 14 cannot be explained by a warp (especially profiles 2 and 5, corresponding to the south of A4 and to B1, since they are close to the minor axis). The warp may however explain some large profiles, such as that observed in knot A3 (profile 4 of Fig. 14) in the SE end of UGC 6697.

The $2 \mathrm{D}$ velocity field provided by the Fabry-Perot data suggests indeed that there is a strong interaction between two galaxies. The peculiar morphology, with a warp to the SE and a tidal tail on the other side, added to the strong velocity jumps observed on the long-slit spectra, are by themselves suggesting an interaction. The FabryPerot, thanks to its full 2D coverage, reveals several isolated blobs of ionized gas with peculiar velocities (as seen at the end of Sect. 3.4). Moreover, this full 2D coverage suggests that there are indeed two intricate velocity structures as can be seen in Fig. 16 where the isovelocity lines have been superposed on the colour coded velocity field. A smaller galaxy appears to be quite distinct from the main galaxy and just under it, in the central part. These two objects are displayed separately in Fig. 17 for which the velocity field was built by following carefully each component as explained in Sect. 3.4 (whereas the velocity field of Fig. 15 only shows the brightest of the two components when there is superposition). The interesting fact is that within each of the two galaxy one can see a gradient revealing rotation. Although the velocity fields are
Table 7. The parameters used to compute the rotation curves of the main and second galaxies from the $2-\mathrm{D}$ velocity field.

\begin{tabular}{lccc}
\hline & main galaxy & second galaxy \\
\hline Systemic velocity & 6680 & 6790 & $\mathrm{~km} \mathrm{~s}^{-1}$ \\
Inclination & 75 & 60 & $\mathrm{deg}$ \\
PA & 135 & 160 & $\mathrm{deg}$ \\
Sector from PA & 20 & 50 & $\mathrm{deg}$ \\
Rotation Center & A4 & south spot of A4 \\
\hline
\end{tabular}

not so well behaved as with regular rotating disks, it must be noted that the isovelocity lines are not as distorted as could be expected with interacting objects. Figure 18 shows the corresponding rotation curves, drawn as if we were dealing with normal disk galaxies. The parameters used for each curve are given in Table 7 . The result must be taken with care however, because of the high inclination of the main body and superposition of both objects. The parameters found for the second galaxy are quite uncertain since its velocity field is rather asymmetric ${ }^{1}$, with the blueshifted side displaying a solid body rotation, whereas the redshifted side rapidly reaches a plateau. Furthermore the signal to noise ratio is rather low for the blueshifted side, meanwhile the redshifted side is mostly absorbed by the foreground galaxy.

Also it is hard to find the rotation center for the second object, nothing looking like a possible nucleus being found in the area where the velocity gradient is maximum. We assumed in fact that a large part of this galaxy is hidden behind the main galaxy and adopted as a rotation center the redder spot of A4 seen on the HST image (Fig. 10). It could be indeed the nucleus of the second galaxy since the rotation curve thus obtained (see Fig. 18) is fairly symmetric, although it is about twice more extended on the northwestern side (note however that the rising part of the curve, beyond 45 arcsec, is not reliable since it is produced by velocity points on the edge of the object, with low signal to noise ratio). It is to be noted that, because of the masking effects from the main galaxy, the PA of the major axis of the second galaxy, given in Table 7, could be off by 20 degrees as well as the inclination of its disk.

In spite of their distinct kinematical behaviour the two objects are almost impossible to clearly identify on the images of Figs. 1-4 and 8. However it seems that the second galaxy not only coincides with the reddest region, but also with the region where the highest metallicity and the strongest extinction is detected.

In conclusion, we tentatively propose a scenario where UGC 6697 is composed of two interacting galaxies which we will identify as "main galaxy" and "second galaxy". The second galaxy, seen through the main galaxy is reddened, and perhaps of higher metallicity. The long NW

\footnotetext{
1 We remark that in the center of UGC 6697 there is a strong consistency between the double velocities separated by $\sim 200 \mathrm{~km} \mathrm{~s}^{-1}$ detected in this work and those derived from CO spectra by Boselli et al. (1994).
} 
extension of the main galaxy (with almost constant recessional velocity) could be a tidal tail due to the gravitational interaction. Using the method proposed by Lequeux (1983) we can estimate the masses of the two interacting galaxies: $M=0.8 R V^{2} / G$, where $M$ is the estimated mass within the radius $R, V$ the rotation velocity at the radius $R$, and $G$ the gravitational constant. The rotation curves (see Fig. 18) indicate the following values, respectively for the main galaxy and for the second galaxy (assuming a distance of $90 \mathrm{Mpc}$ ): $340 \mathrm{~km} \mathrm{~s}^{-1}$ at $100 \operatorname{arcsec}(42 \mathrm{kpc})$ and $300 \mathrm{~km} \mathrm{~s}^{-1}$ at $45 \operatorname{arcsec}(18 \mathrm{kpc})$; hence the estimated masses of $9 \times 10^{11}$ solar masses and $3 \times 10^{11}$ solar masses respectively. Of course there are large uncertainties on these values because the total extent of the rotation curve of the main galaxy toward the high velocities is probably biased by what appears to be a tidal tail seen edge on (which may lead to overestimate the mass by a factor 2 , although in the above estimate we did not include the two outermost $\mathrm{H}_{\alpha}$ emission areas seen in Fig. 15), and the inclination of the second galaxy is rather uncertain. Anyway we can affirm that both objects are quite massive galaxies, the main one being about 2 to 3 times heavier than its companion.

Acknowledgements. G. G. wishes to thank his students D. Carbone, A. Colombo, A. Gualandris, D. Malesani, D. Marchesini, F. Padoan, C. Pagani, G. Toso for their precious contribution during the data reduction of the Loiano spectra. C. Bonfanti is warmly acknowledged for his contribution in the reduction and interpretation of the low dispersion spectrum. M. M. thanks his student, O. Garrido, for her contribution to the data reduction of OHP Fabry-Perot observations. We thank the referee, A. Bosma, for his criticism which contributed to improving this work.

\section{References}

Amram, P., Mendes de Oliveira, C., Boulesteix, J., \& Balkowski, C. 1998, A\&A, 330, 881

Boselli, A., Gavazzi, G., Combes, F., Lequeux, J., \& Casoli, F. 1994, A\&A, 285, 69

Boselli, A., Gavazzi, G., Donas, J., \& Scodeggio, M. 2001, AJ, 121,753

Dickey, J., \& Gavazzi, G. 1991, ApJ, 373, 347

Dopita, M., et al. 2000, ApJ, 542, 224

Edmunds, M. G., \& Pagel, B. E. J. 1984, MNRAS, 211, 507

Gavazzi, G. 1978, A\&A, 69, 355

Gavazzi, G., Tarenghi, M., Jaffe, W., Butcher, H., \& Boksenberg, A. 1984, A\&A, 137, 235 (G84)

Gavazzi, G., \& Jaffe, W. 1985, ApJL, 294, L89

Gavazzi, G., \& Jaffe, W. 1987, A\&A, 186, L1

Gavazzi, G. 1989, ApJ, 346, 59

Gavazzi, G., Contursi, A., Carrasco, L., et al. 1995, A\&A, 304, 325 (G95)

Gavazzi, G., Zibetti, S., Boselli, A., et al. 2001, A\&A, in press

Lemaitre, G., Kohler, D., Lacroix, D., Meunier, J., \& Vin, A. 1990, A\&A, 228, 540

Lequeux, J. 1983, A\&A, 125, 394

Merritt, D. 1983, ApJ, 264, 24

Moore, B., Katz, N., Lake, G., Dressler, A., \& Oemler, A. 1996, Nature, 379, 613

Moore, B., Lake, G., \& Katz, N. 1998, ApJ, 495, 139

Nulsen, P. 1982, MNRAS, 198, 1007 